\title{
Emission of X-Rays through the Recombination in the Collision of F-like Ions with Energetic Free Electrons
}

\author{
H. Hanafy and F. Shahin \\ Physics Department, Faculty of Science, Beni-Suef University, Egypt.
}

In thermal plasma, there are many recombination processes which are responsible for ionization balance and self-cooling (emission of $x$-rays) of thermal plasmas (about $10^{7}{ }^{0} \mathrm{~K}$ ). The resonant (indirect) recombination of electrons with positive ions is often the dominant mode overall the direct recombination processes. The radiative recombination ( $R R$ ) process is relatively large for low-energy of the incident electrons. As the continuum (free) energy increases, the dielectronic recombination (DR) starts to dominate. However, the three-body recombination (TBR) is important only for the high Rydberg states (HRS) with $n>20$; where $n$ is the principal quantum number of the captured electrons in positive ions. Recently, it is well-known that, the accurate values of $D R$ rates, together with ionization rates are required for precise determination of the characteristics of astrophysical and laboratory thermal plasmas. In the present work, the DR cross sections $\left(\sigma^{D R}\right)$ and rate coefficients $\left(\alpha^{D R}\right)$ are calculated for F-like $\mathrm{Al}^{4+}, \mathrm{Ar}^{9+}$ and $\mathrm{Cr}^{15+}$ ions with L-shell excitation. The intershell $(\Delta n \neq 0)$ transitions are taken into consideration. However, the intra-shell $(\Delta n=0)$ transitions are disregarded. It is found that, both $\sigma^{D R}\left(\mathrm{~cm}^{2}\right)$ and $\alpha^{D R}$ $\left(\mathrm{cm}^{3} / \mathrm{sec}\right)$ for L-shell excitations, with $\Delta n=0$, are much larger than those for $K$ shell excitations. The isoelectronic trends of both $\sigma^{D R}$ and $\alpha^{D R}$ with some atomic parameters such as effective charge $\left(Z_{\text {eff }}\right)$ and atomic number $(Z)$ of $F$-like ions are investigated.

\section{Introduction:}

In the collisions of energetic electrons with positive ions, a continuum electron may be captured by a positively charged ion having bound electrons with a simultaneous excitation. This capture process may create resonance (doubly-excited) states. When these d-states stabilize by emission of radiation, the process is known as dielectronic recombination (DR). The DR process is one of the recombination processes which imbalance with the ionization in hot plasma. In addition, it calls self cooling of the thermal plasma. 
In the last two decades, many theoretical [1-5] and experimental [6-9] works have been done to understand the DR process and the effect of different angular momenta coupling schemes on the calculation of its rate coefficients. Although of such extensive and intensive studies, some works are still required to simplify the existing lengthy method of calculations.

The resonance d-states are usually formed from the ground (i) state by a capture of a free electron which, in turn, causes inter-shell $(\Delta \mathrm{n} \neq 0)$ or intra-shell $(\Delta \mathrm{n}=0)$ excitations. The excitation energies for inter-shell excitations are much larger than that for intra-shell excitations. Thus, intra-shell excitations dominate only at low temperatures and for light ions. However, the inter-shell excitations are interesting at high temperatures and for heavy ions. The present work deals with the calculation of DR cross sections and rates for L-shell excitation with $\Delta \mathrm{n} \neq 0$ transitions only. The calculations of DR cross sections and rates for ions with open shells in their ground states are more complicated than that the ions with closed shells. Thus, the adapted angular momentum average (AMA) scheme is employed in the present calculations to minimize the size of the DR calculations to a manageable size.

Many approximations are recently introduced to reduce the size of work. For example, the empirical formula which was constructed for ironions[10] within uncertainty of $\pm 15 \%$. such empirical formulae are independent of the detailed information about d-states. However, the accuracy required for the atomic data for modeling purpose can not be very high.

In addition, the method of calculation of DR rates has been modified to compute the DR rates depending on the final states reached by radiative decays [11]. Moreover, the DR rates are also calculated for ions with initial excited states [13].

In order to generate the DR rates for F-like ions with L-shell excitation, the AMA scheme is utilized to compute the Auger $\left(\overline{\mathrm{A}}_{\mathrm{a}}\right)$ and radiative $\left(\overline{\mathrm{A}}_{\mathrm{r}}\right)$ transition probabilities which are required in these DR calculations. It has to be noted that, the calculations of too many $\overline{\mathrm{A}}_{\mathrm{a}}$ 's and $\overline{\mathrm{A}}_{\mathrm{r}}$ 's associated with each $\mathrm{d}$ state may be possible only in AMA scheme for ions with open L-shell in their ground states. Although AMA scheme may give an overestimate for DR rates corresponding to some d-states, the total DR rates are still reliable with uncertainty 5-10\%. Thus, the trends of DR rates in this adapted AMA scheme are usually unaltered if other coupling schemes are used. 


\section{Method of Calculations:}

The DR cross sections $\left(\sigma^{\mathrm{DR}}\right)$ and rate coefficients $\left(\bar{\alpha}^{\mathrm{DR}}\right)$ are calculated in the isolated resonance approximation (IRA) for each d-state using the following two formulas:

For DR cross sections, we have:

$$
\sigma^{D R}=C_{o} V_{a}(i \rightarrow d) w(d) \frac{1}{e_{c} \Delta e_{c}}
$$

where the constant is estimated as : $\mathrm{C}_{0}=2.68 \times 10^{-32} \mathrm{~cm}^{2} \cdot \mathrm{sec}$ For DR rates, we have:

$$
\bar{\alpha}^{\mathrm{DR}}(\mathrm{i} \rightarrow \mathrm{d})=\left[\frac{4 \pi R y}{\mathrm{k}_{\mathrm{B}} \mathrm{T}_{\mathrm{e}}}\right]^{3 / 2} \cdot \mathrm{a}_{\mathrm{o}}^{3} \cdot \overline{\mathrm{V}}_{\mathrm{a}}(\mathrm{i} \rightarrow \mathrm{d}) \cdot \bar{\omega}(\mathrm{d}) \cdot \exp \left(-\mathrm{e}_{\mathrm{c}} / \mathrm{k}_{\mathrm{B}} \mathrm{T}_{\mathrm{e}}\right)
$$

The total rate coefficient corresponding to a given ion with atomic number $(\mathrm{Z})$, the electron temperature $(\mathrm{T})$ and the degree of ionization (q) can be written as:

$$
\bar{\alpha}^{\mathrm{DR}}(\mathrm{Z}, \mathrm{q}, \mathrm{T}, \mathrm{i})=\sum_{d} \bar{\alpha}_{\mathrm{i} \rightarrow \mathrm{d}}^{\mathrm{DR}}(\mathrm{Z}, \mathrm{q}, \mathrm{T})
$$

where, $\mathrm{V}_{\mathrm{a}}(\mathrm{i} \rightarrow \mathrm{d})$ is the radiationless capture probability which is obtained using Auger rate from d-state to i-state and $\bar{\omega}(\mathrm{d})$ is the fluorescence yield.

The Auger rate for two equivalent electrons $\left(n_{3} \ell_{3}=n_{4} \ell_{4}\right)$ in d-states is given by

$$
\overline{\mathrm{A}}_{\mathrm{a}}=\frac{\left(h_{1}+1\right) \cdot\left(4 \ell_{3}+2-h_{3}\right) \cdot\left(4 \ell_{3}+1-h_{3}\right)}{\left(4 \ell_{3}+2\right) \cdot\left(4 \ell_{3}+1\right)} \overline{\mathrm{A}}_{\mathrm{a}}^{(0)}
$$

In addition, the Auger rate for nonequivalent electrons $\left(n_{3} \ell_{3} \neq n_{4} \ell_{4}\right)$ is given by

$$
\overline{\mathrm{A}}_{\mathrm{a}}=\frac{\left(h_{1}+1\right) \cdot\left(4 \ell_{3}+2-h_{3}\right) \cdot\left(4 \ell_{4}+2-h_{4}\right)}{\left(4 \ell_{3}+2\right) \cdot\left(4 \ell_{4}+2\right)} \overline{\mathrm{A}}_{\mathrm{a}}^{(0)}
$$

The quantities $n, \ell$ represent the principal and orbital quantum numbers of the active electrons in d-state. $h_{1}, h_{2}$ and $h_{3}$ are the number of holes in the orbits involved in Auger transitions. 
The radiationless capture probability $\left(\overline{\mathrm{V}}_{\mathrm{a}}\right)$ is then obtained from $\overline{\mathrm{A}}_{\mathrm{a}}\left(\mathrm{d} \rightarrow \mathrm{i}, \ell_{\mathrm{c}}\right)$ using the formula

$$
\bar{V}_{a}(i \rightarrow d)=\left(\frac{g_{d}}{g_{e} g_{i}}\right) \sum_{l} \bar{A}_{a}\left(d \rightarrow i, \ell_{c}\right)
$$

where $g_{d}, g_{i}$ and $g_{e}$ are the statistical weights for the states $d, i$ and the incident free electron respectively.

The weighted (modified) radiative transition probability may be written as

$$
\bar{A}_{r}(d \rightarrow f)=\frac{N_{d}\left\lfloor 4 \ell_{f i}+2-N_{f i}\right\rfloor}{4 \ell_{f i}+2} \bar{A}_{r}^{(0)}
$$

where $\overline{\mathrm{A}}_{\mathrm{r}}^{(0)}$ refers to the single electron radiative transition rate. $\mathrm{N}_{\mathrm{d}}$ represents the number of electrons in the d-state. $\mathrm{N}_{\mathrm{fi}}$ stands for the number of electrons in the final (f) state initially. $\ell_{\text {if }}$ is the orbital quantum number of the f-state initially.

The total resonant width of a d-state is the sum of all possible radiative and Auger widths, i.e.,

$$
\bar{\Gamma}(\mathrm{d})=\bar{\Gamma}_{\mathrm{a}}(\mathrm{d})+\bar{\Gamma}_{\mathrm{r}}(\mathrm{d})
$$

$\bar{\Gamma}_{\mathrm{a}}(\mathrm{d})$ and $\bar{\Gamma}_{\mathrm{r}}(\mathrm{d})$ are the Auger and radiative widths.

where $\bar{\Gamma}_{\mathrm{a}}(\mathrm{d})$ is the sum of all Auger decay rates of the d-state and may be written as

$$
\bar{\Gamma}_{a}(d)=\left[\sum_{i, \ell_{c}} \bar{A}_{a}\left(d \rightarrow i, \ell_{c}\right)+\sum_{j, \ell_{c}^{\prime}} \bar{A}_{a}\left(d \rightarrow j, \ell_{c}^{\prime}\right)\right]
$$

and $\bar{\Gamma}_{\mathrm{r}}(\mathrm{d})$ is the sum of all radiative decay rates of the d-state and may be written as:

$$
\bar{\Gamma}_{\mathrm{r}}(\mathrm{d})=\sum_{f, f^{\prime}} \overline{\mathrm{A}}_{\mathrm{r}}\left(\mathrm{d} \rightarrow \mathrm{f}, \mathrm{f}^{\prime}\right)
$$

The fluorescence yield [ $\bar{\omega}(\mathrm{d})]$ is defined by

$$
\bar{\omega}(\mathrm{d})=\bar{\Gamma}_{\mathrm{r}}(\mathrm{d}) /\left[\bar{\Gamma}_{a}(\mathrm{~d})+\bar{\Gamma}_{r}(\mathrm{~d})\right]
$$




\section{Results and Discussion:}

The DR cross sections $\sigma^{\mathrm{DR}}$ and DR rates $\bar{\alpha}^{\mathrm{DR}}$ for $\mathrm{F}$-like $\mathrm{Al}^{4+}, \mathrm{Ar}^{9+}$ and $\mathrm{Cr}^{15+}$ ions are calculated in isolated resonance approximations (IRA). In these calculations, the basic parameters such as radiative $\left(\overline{\mathrm{A}}_{\mathrm{r}}\right)$ and Auger $\left(\overline{\mathrm{A}}_{\mathrm{a}}\right)$ rates as well as fluorescence yield $\bar{\omega}(\mathrm{d})$ are computed in details in AMA scheme as a first step. The wavefunctions which are needed in our calculations are obtained from the Hartree-Fock program (Fischer code). The matrix program (Hahn's code) is employed to generate all $\mathrm{A}_{\mathrm{a}}$ 's and $\mathrm{A}_{\mathrm{r}}$ 's rates.

\section{The DR Cross sections:}

The DR cross sections for F-like $\mathrm{Al}^{4+}, \mathrm{Ar}^{9+}$ and $\mathrm{Cr}^{15+}$ ions are calculated and presented in Fig. (1) including both $2 \mathrm{~s}-$ and $2 \mathrm{p}$ - excitations. It is clear that, for $\mathrm{Al}^{4+}$, the DR cross sections lie between $5 \mathrm{Ry}$ and $12 \mathrm{Ry}$ as the incident free electron energies. The DR cross sections are represented for this ion with many closed peaks. However, there is an isolated peak at 5.85 Ry and the value of this peak is $2.84 \times 10^{-22} \mathrm{~cm}^{2}$. The peaks in this ion, part (a), are more crowded than the other studied ions. This reflects the nature of the doubly excited stated formed in case of $\mathrm{Al}^{4+}$. In addition, the calculation of the DR cross sections must be done carefully for each individual doubly excited states for ions with low degree of ionization. In Fig. (1b), the DR cross sections are presented for $\mathrm{Ar}^{9+}$ contain about seven pronounced peaks. The largest peak lies at $18.6 \mathrm{Ry}$ with a peak value of $10.74 \times 10^{-22} \mathrm{~cm}^{2}$. These peaks are slightly separated

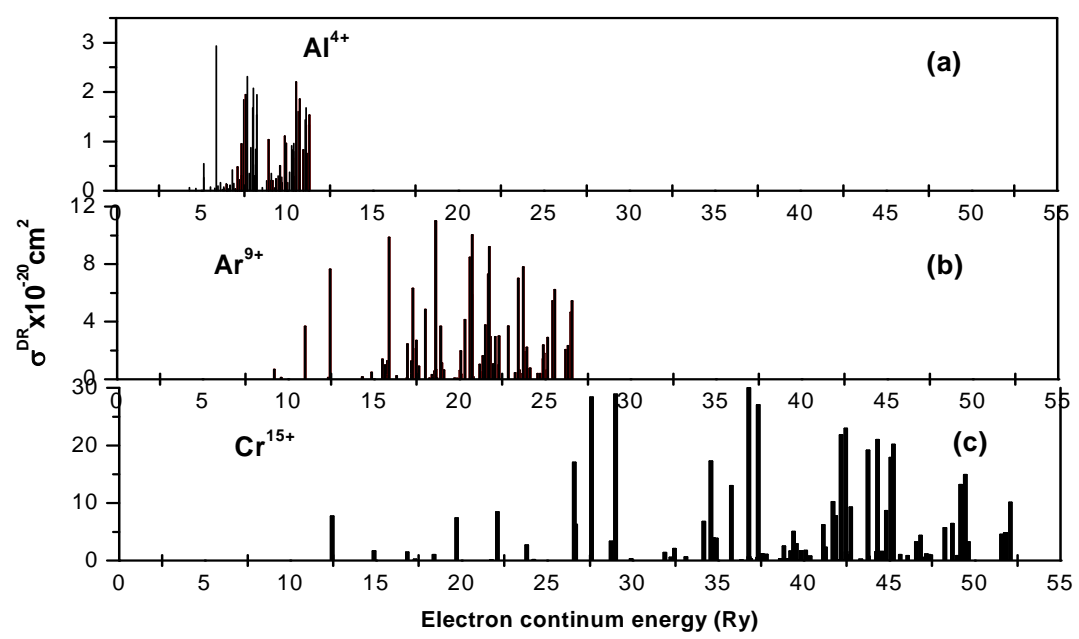

Fig. (1): Total $\sigma^{D R} \times 10^{-20} \mathrm{~cm}^{2}$ for F-like $\left(\mathrm{Al}^{4+}, \mathrm{Ar}^{9+}\right.$ and $\left.\mathrm{Cr}^{15+}\right)$ ions with L-shell $\left(2 \mathrm{~s}+2 \mathrm{p}\right.$ ) excitation. (a) is for $\mathrm{Al}^{4+}$, (b) is for $\mathrm{Ar}^{9+}$ and (c) is for $\mathrm{Cr}^{15+}$.

\section{The DR Rates:}


The DR rates for $\mathrm{Al}^{4+}, \mathrm{Ar}^{9+}$ and $\mathrm{Cr}^{15+}$ ions with $2 \mathrm{~s}$ - and $2 \mathrm{p}$ - shell excitations are calculated in IRA approximation using equation (1) at different thermal energies (kT's) of the continuum electrons.

The variation of DR rates ( $\bar{\alpha}^{\mathrm{DR}}$,s) with the thermal energy of the continuum electron $(\mathrm{kT})$ for $\mathrm{Cr}^{15+}$ with $2 \mathrm{~s}$ - and $2 \mathrm{p}$ - shell excitation is drawn in Fig. (2). It is clear that $\bar{\alpha}^{\mathrm{DR}}$ varies smoothly with kT. All curves of DR rates in Fig. (2) are symmetric around the peak values. This reflects the nature of the Maxwellian distribution for the velocities of free electrons. From previous studies, the DR rates for all isoelectronic sequences vary smoothly with kT except for K-like ions [13]. The dominant DR rates values in the L-shell excitation is observed from the doubly excited states $1 \mathrm{~s}^{2} 2 \mathrm{~s}^{2} 2 \mathrm{p}^{4} 3 \mathrm{dn} l$ which is larger than that from the doubly excited states $1 \mathrm{~s}^{2} 2 \mathrm{~s} 2 \mathrm{p}^{5} 3 \mathrm{dn} l$. Where $\mathrm{n}$ is the principle quantum number $(\mathrm{n}=3,4,5, \ldots \ldots)$ and $l$ is the orbital quantum number $(l=\mathrm{s}, \mathrm{p}, \mathrm{d}$, and $\mathrm{f})$. The contribution of the doubly excited states $1 \mathrm{~s}^{2} 2 \mathrm{~s}^{2} 2 \mathrm{p}^{4} 3 \mathrm{sn} l$, $1 \mathrm{~s}^{2} 2 \mathrm{~s}^{2} 2 \mathrm{p}^{4} 3 \mathrm{pn} l, 1 \mathrm{~s}^{2} 2 \mathrm{~s} 2 \mathrm{p}^{5} 3 \mathrm{sn} l$ and $1 \mathrm{~s}^{2} 2 \mathrm{~s} 2 \mathrm{p}^{5} 3 \mathrm{pn} l$ are small.

In addition, the DR rates for 2s-excitation are much larger than that for $2 p$-excitation which changed as the degree of ionization increases and became $2 \mathrm{p}$-excitation are much larger than that for $2 \mathrm{~s}$-excitation. This indicates that the DR process is more efficient for outer shell excitations. This indication is consistent with the fact that the DR rates with L-shell excitation are few hundreds larger than that with K-shell excitation. It has to be noted that all transitions from the $2 p$-shell are inter-shell excitations. However, the $2 s-$ excitation includes both inter-shell and intra-shell excitations. In fact, this work involves the inter-shell excitations only for the 2 s-orbital. The DR rates for $2 \mathrm{~s}-$ and $2 p$-shell excitations as well as the total DR rates for L-shell $(2 s+2 p)$ are presented in Fig. (2)., for $\mathrm{Cr}^{15+}$ ion.

The total DR rates for $\mathrm{Al}^{4+}, \mathrm{Ar}^{9+}$ and $\mathrm{Cr}^{15+}$ ions with L-shell excitation are calculated using the same method in the IRA approximation. The values of the total DR rates for $\mathrm{F}$-like $\mathrm{Al}^{4+} \mathrm{Ar}^{9+}$, and $\mathrm{Cr}^{15+}$ ions are presented together in Figure (3). It is obvious from this figure that the peak positions of the ions are shifted towards high energy values as the atomic number $(Z)$ of the ions increases. In addition, the values of the peaks increase also with increasing $Z$. Specifically, the peak values are $4.33 \times 10^{-11} \mathrm{~cm}^{3} / \mathrm{sec}, 17.41 \times 10^{-11} \mathrm{~cm}^{3} / \mathrm{sec}$ and $43.5 \times 10^{-11} \mathrm{~cm}^{3} / \mathrm{sec}$ for $\mathrm{Al}^{4+}, \mathrm{Ar}^{9+}$ and $\mathrm{Cr}^{15+}$ respectively. It is noted that the DR rate becomes broader curve as the atomic number $(\mathrm{Z})$ of the ion increases.

Thus, the calculation of the DR rates for these states only in other coupling schemes may be used to predict reliable values of the total DR rates 
for these ions without loosing any physical information. This prediction will simplify the calculations and avoid the complexity exist in other coupling schemes such as LS-coupling and JJ-coupling schemes of angular momenta of open shells
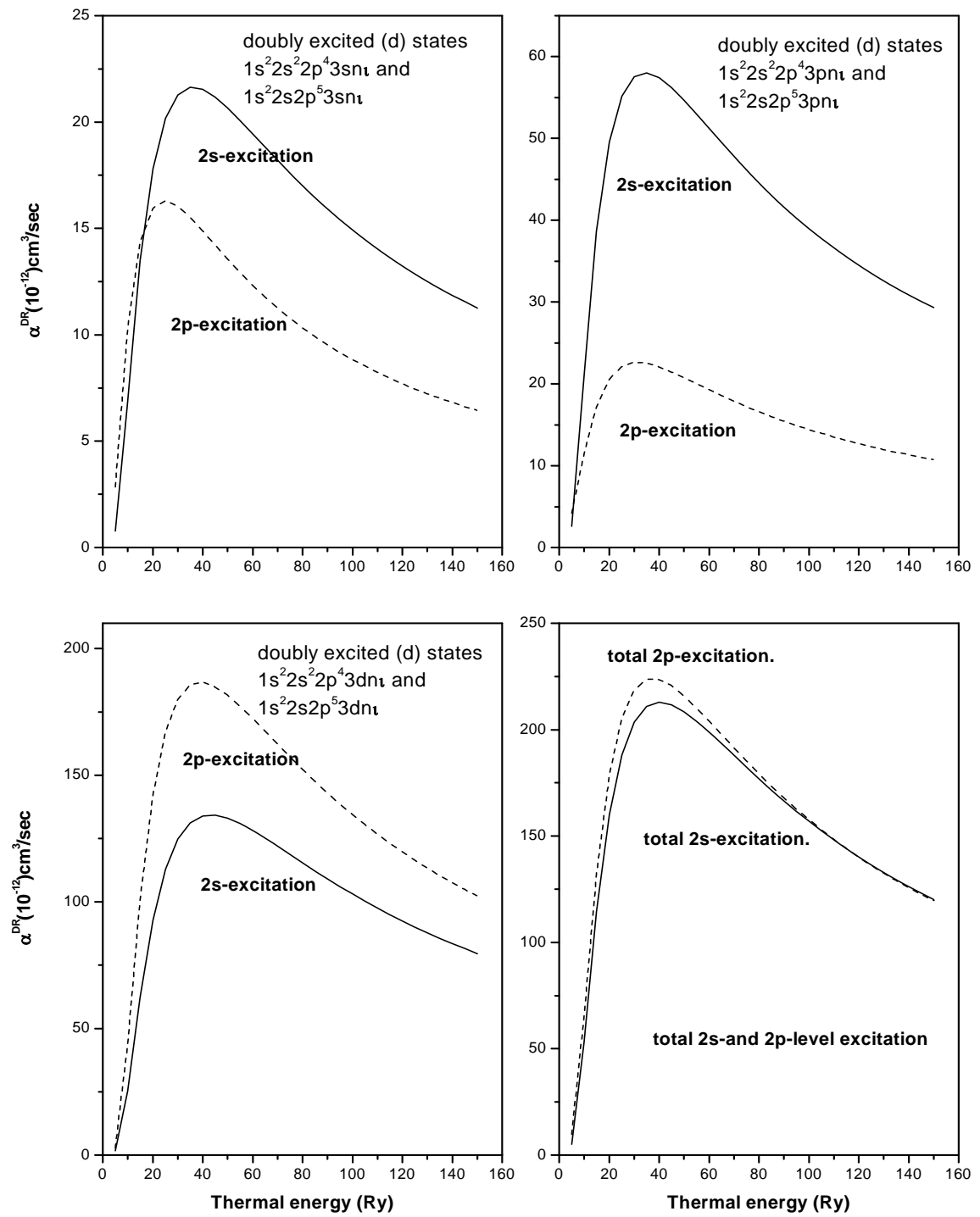

Fig. (2): DR rates, $\alpha^{D R} \times 10^{-12} \mathrm{~cm}^{3} / \mathrm{sec}$ for $\mathrm{Cr}^{15+}$ with L-shell (2s+2p) excitations. 


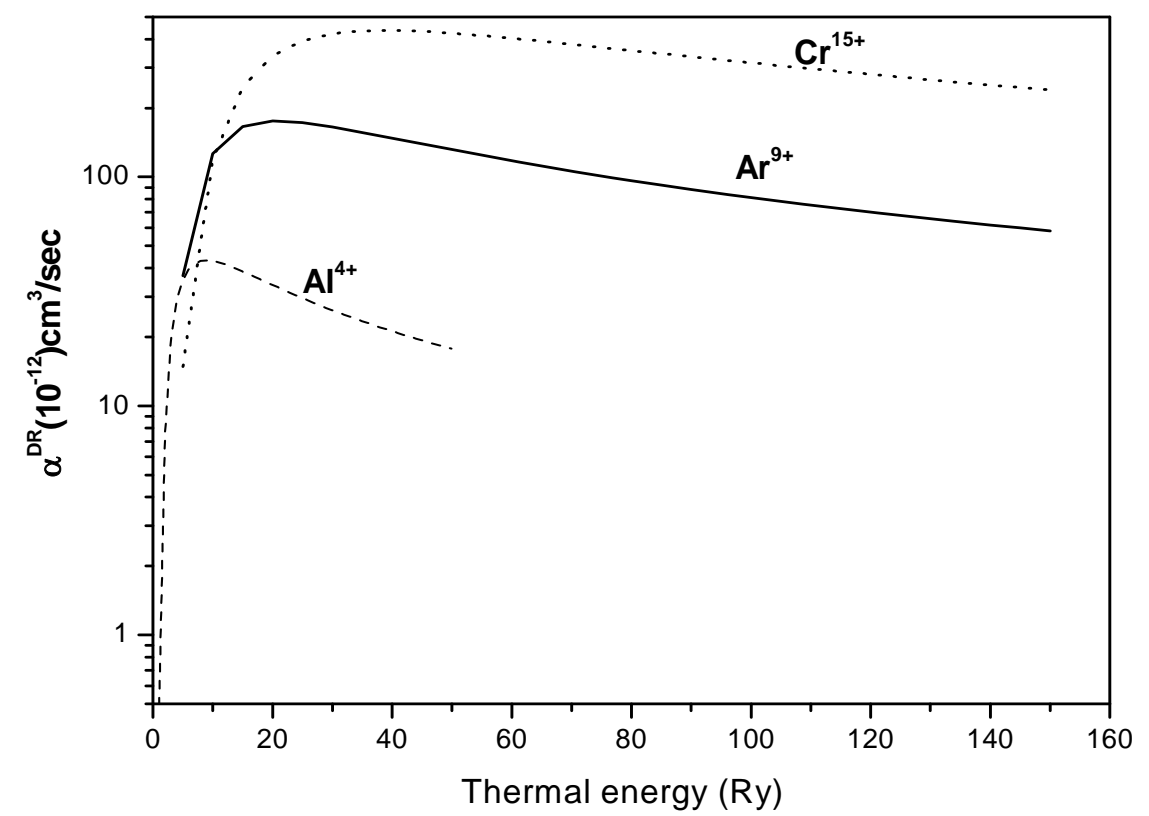

Fig. (3): Total DR rates, $\alpha^{D R} \times 10^{-12} \mathrm{~cm}^{3} / \mathrm{sec}$ for $\mathrm{Al}^{4+}, \mathrm{Ar}^{9+}$ and $\mathrm{Cr}^{15+}$ with L-shell $(2 s+2 p)$ excitation.

\section{The Isoelectronic Trends of the DR Rates:}

To investigate the isoelectronic trends of the DR rates with L-shell excitation, we can use Table (1) and Fig. (3).

Table (1): The atomic number $(\mathrm{Z})$, the effective charge $\left(\mathrm{Z}_{\mathrm{eff}}\right)$ and the peak value of the total DR-rate with L-shell excitation in units of $10^{-11} \mathrm{~cm}^{3} / \mathrm{s}$ for each F-like ion.

\begin{tabular}{|c|c|c|c|}
\hline Ion & $Z$ & $Z_{\text {eff }}$ & $\begin{array}{c}\text { Peak value } \\
\left(10^{-11} \mathrm{~cm}^{3} / \mathrm{s}\right)\end{array}$ \\
\hline $\mathrm{Al}^{4+}$ & 13 & 8.5 & 4.33 \\
\hline $\mathrm{Ar}^{9+}$ & 18 & 13.5 & 17.41 \\
\hline $\mathrm{Cr}^{15+}$ & 24 & 19.5 & 43.5 \\
\hline
\end{tabular}

As mentioned previously, the peak values of $\bar{\alpha}_{\text {tot }}^{\mathrm{DR}}$ increase with increasing $Z$ of the ion. This helps to deduce the dependence of $\bar{\alpha}_{\text {tot }}^{\mathrm{DR}}$ on the effective charge $\left(Z_{\text {eff }}\right)$ of the ion at peak. The effective charge in atomic collisions has the form $Z_{\text {eff }}=(Z+q) / 2$ where $Z$ and $q$ are the atomic number and 
the degree of ionization of the ion, respectively. To show such dependence, we proceed as follows:

$$
\begin{aligned}
& \frac{\bar{\alpha}_{\mathrm{tot}}^{\mathrm{DR}}\left(\mathrm{Ar}^{9+}\right)}{\bar{\alpha}_{\mathrm{tot}}^{\mathrm{DR}}\left(\mathrm{Al}^{4+}\right)}=\frac{17.41 \times 10^{-11}}{4.33 \times 10^{-11}}=4.02 \\
& {\left[\frac{\mathrm{Z}_{\mathrm{eff}}\left(\mathrm{Ar}^{9+}\right)}{\mathrm{Z}_{\mathrm{eff}}\left(\mathrm{Al}^{4+}\right)}\right]^{3.04}=\left[\frac{13.5}{8.5}\right]^{3.04}=4.02,} \\
& {\left[\frac{\mathrm{Z}\left(\mathrm{Ar}^{9+}\right)}{\mathrm{Z}\left(\mathrm{Al}^{4+}\right)}\right]^{4.28}=\left[\frac{18}{13}\right]^{4.28}=4.02}
\end{aligned}
$$

This means that the peak values of $\bar{\alpha}_{\text {tot }}^{\mathrm{DR}}$ varies as $Z_{\text {eff }}^{3.04}$ and $Z^{4.28}$ for ions with $4 \leq q<10$. Similarly, we find that the DR rate increases approximately as $Z^{3.17}$ and $Z_{\text {eff }}^{2.48}$ for ions with $9 \leq \mathrm{q}<16$. However, the ratios are dependent on the effective charge $\left(Z_{\text {eff }}\right)$ and the atomic charge $(Z)$. The DR rates are useful in the isoelectronic trend studies. Where, the peak of the DR rates shifts to a higher energy as the degree of ionization increases. Moreover, $\bar{\alpha}^{\mathrm{DR}}$ depends mainly on the effective charge $\left(Z_{\text {eff }}\right)$ and the nuclear charge $(Z)$.

\section{Conclusion:}

All DR rates change smoothly with the thermal energy (kT) of the continuum electron. All curves of DR rates are symmetric around the peak values. This situation reflects the nature of the Maxwellian distribution for the velocities of free electrons. The peak value of $\bar{\alpha}^{\mathrm{DR}}$ for $2 \mathrm{p}$-excitation is larger than that of $2 \mathrm{~s}$-excitation by factors of $4.4 \times 10^{-11}, 2.3 \times 10^{-11}$ and $1.8 \times 10^{-11}$ $\mathrm{cm}^{3} / \mathrm{sec}$ for $\mathrm{Al}^{4+}, \mathrm{Ar}^{9+}$ and $\mathrm{Cr}^{15+}$ respectively. Therefore, DR manifests itself as a more efficient mechanism for outer shells. The position of $\bar{\alpha}_{\text {tot }}^{\mathrm{DR}}$ peak is shifted to a higher value of $\mathrm{kT}$ as the atomic number $(\mathrm{Z})$ of the ions increases. Specifically, for L-shell $(2 \mathrm{~s}+2 \mathrm{p})$ excitation, $\bar{\alpha}_{\mathrm{tot}}^{\mathrm{DR}}$ is peaked at $\mathrm{kT}=8.94,20.7$, and 38.5 $\mathrm{Ry}$ for $\mathrm{Al}^{4+}, \mathrm{Ar}^{9+}$ and $\mathrm{Cr}^{15+}$ respectively. The peak value of $\bar{\alpha}_{\text {tot }}^{\mathrm{DR}}$ increases with the increase in nuclear charge $(Z)$ of the ions in the same isoelectronic sequence. Specifically, for L-shell excitation, the peak values of $\bar{\alpha}_{\text {tot }}^{\mathrm{DR}}$ are 4.33, 17.41, and $43.5 \times 10^{-11} \mathrm{~cm}^{3} / \mathrm{sec}$ for $\mathrm{Al}^{4+}, \mathrm{Ar}^{9+}$ and $\mathrm{Cr}^{15+}$ respectively.

\section{References:}


1. M.S. Pindzola and D.C. Griffin, Phys. Rev. A 56, 1654 (1997).

2. Y. Hahn, Adv. Atom. Mol. Phys. 21, 123 (1985).

3. J. Gau and Y. Hahn, JQSRT 23, 121 (1980).

4. Y. Hahn et al., Phys. Rev. A36, 576 (1987).

5. G. Omar, Egypt. J. of Phys., 31, 235 (2000).

6. W. Zong et al., Phys. Rev. A 56, 386 (1997).

7. D. R. DeWitt et al., Phys. Rev. A 53, 2327 (1996).

8. R. Schuch et al., Nucl. Inst. and Meth. in Phys. Res. B 79, 59 (1993).

9. P. Glans et al., Nucl. Instr. and Methods B 154, 97 (1999).

10. Y. Hahn, JQSRT 41, 315 (1989).

11. G. Omar et al., Phys. Rev. E 62, 4096 (2000).

12. G. Omar et al., Phys. Rev. E 63, 46407 (2001).

13. G. Omar et al., Phys. Rev. A4 